IFT-P. 2010

August, 2010

\title{
Generating Erler-Schnabl-type Solution for Tachyon Vacuum in Cubic Superstring Field Theory
}

\author{
E. Aldo Arroyd* \\ Instituto de Física Teórica, UNESP - Universidade Estadual Paulista \\ Caixa Postal 70532-2 \\ 01156-970 São Paulo, SP, Brasil
}

\begin{abstract}
We study a new set of identity-based solutions to analyze the problem of tachyon condensation in open bosonic string field theory and cubic superstring field theory. Even though these identity-based solutions seem to be trivial, it turns out that after performing a suitable gauge transformation, we are left with the known Erler-Schnabl-type solutions which correctly reproduce the value of the D-brane tension. This result shows explicitly that how a seemingly trivial solution can generate a non-trivial configuration which precisely represents to the tachyon vacuum.
\end{abstract}

*aldohep@ift.unesp.br 


\section{Contents}

1 Introduction

2 Simple analytic solution in the bosonic case

2.1 Erler-Schnabl's solution . . . . . . . . . . . . . . . . . 6

2.2 The identity-based solution and gauge transformation . . . . . . . . . . . 7

2.3 Two-parameter family of solutions . . . . . . . . . . . . . . . . . 9 9

3 Simple analytic solution in the superstring case 12

3.1 The identity-based solution and gauge transformation . . . . . . . . . . 12

3.2 Gorbachev's solution and its vacuum energy . . . . . . . . . . . . . 13

3.3 Two-parameter family of solutions in the superstring case . . . . . . . . . 15

4 Summary and discussion $\quad 16$

A Derivation of the identity-based solution and gauge transformation 17

\section{Introduction}

Recently it was found a simple analytic solution for tachyon condensation in open bosonic string field theory [1] as well as in the modified cubic superstring field theory [2]. The main feature of these solutions is that, rather than a discrete sum, the solutions (which we refer as the Erler-Schnabl-type solutions) can be written as a continuous integral over wedge states, where no regularization or phantom term is required. Since these solutions do not require the presence of the phantom term, in contrast to the $\mathcal{B}_{0}$ gauge solutions $[3,4,15,6,7,8,19,10,11,12,13,14$, the computation of the value of the D-brane tension is very straightforward.

It would be interesting to find a similar Erler-Schnabl-type solution in the case of Berkovits WZW-type superstring field theory [15]. Since the action for this theory is a nonpolynomial one, the issue for finding the tachyon vacuum solution and the computation of the value of the D-brane tension seems to be highly cumbersome. Therefore, in order to find some clues for this challenging puzzle, we should analyze the problem in a relatively simple formulation of open superstring field theory.

In the literature, there is an old formulation of open superstring field theory, namely the modified cubic superstring field theory [16, 17]. The action in this formulation is cubic 
(instead of the non-polynomial action given by Berkovits [15])

$$
S=-\frac{1}{g^{2}}\left[\frac{1}{2}\langle\langle\Phi, Q \Phi\rangle\rangle+\frac{1}{3}\langle\langle\Phi, \Phi * \Phi\rangle\rangle\right],
$$

where $Q$ is the BRST operator, * stands for Witten's star product [18], and the inner product $\langle\langle\cdot, \cdot\rangle\rangle$ is the standard BPZ inner product with the difference that we must insert the operator $Y_{-2}$ at the open string midpoint. The operator $Y_{-2}$ can be written as the product of two inverse picture changing operators $Y_{-2}=Y(i) Y(-i)$, where $Y(z)=-\partial \xi e^{-2 \phi} c(z)$. The string field $\Phi$ which has ghost number 1 and picture number 0 belongs to the small Hilbert space of the first-quantized matter+ghost open Neveu-Schwarz superstring theory.

As stated in Sen's first conjecture [19, 20, 21], the classical open string field equation of motion should admit a Poincaré invariant solution $\Phi \equiv \Psi$ which is identified as the tachyon vacuum with no D-branes. This statement means that the energy density of the true vacuum found by solving the equation of motion should be equal to minus the tension of the D-brane. Since the energy density of a static configuration is minus the action, for the case of the cubic action, Sen's conjecture can be summarized as follows

$$
\frac{1}{g^{2}}\left[\frac{1}{2}\langle\langle\Psi, Q \Psi\rangle\rangle+\frac{1}{3}\langle\langle\Psi, \Psi * \Psi\rangle\rangle\right]=-\frac{1}{2 \pi^{2} g^{2}} .
$$

The string field equation of motion and Sen's conjecture allow us to fix the kinetic term,

$$
\frac{\pi^{2}}{3}\langle\langle\Psi, Q \Psi\rangle\rangle=-1
$$

In this paper, we propose a new prescription for generating a string field $\Psi$, in cubic string field theories [16, 17, 18, which satisfies the equation of motion $Q \Psi+\Psi * \Psi=0$ and represents to the tachyon vacuum. The procedure of our prescription follows two steps: (i) find a naive identity-based solution [22] of the string field equation of motion, and (ii) perform a gauge transformation [23] over the identity-based solution such that the resulting string field, consistently, represents to the tachyon vacuum 1 .

In order to find identity-based solution 2 to the string field equations of motion, we are going to use a basis similar to the one used in [3] with the difference that in our case the operators are inserted on the identity string field $\mathcal{I}^{3}$. For instance in the case of open bosonic string field theory, to find a solution based on the identity string field, we should use the ansatz

$$
\Psi=\sum_{n, p} f_{n, p} U_{1}^{\dagger} U_{1} \hat{\mathcal{L}}^{n} \tilde{c}_{p}|0\rangle+\sum_{n, p, q} f_{n, p, q} U_{1}^{\dagger} U_{1} \hat{\mathcal{B}} \hat{\mathcal{L}}^{n} \tilde{c}_{p} \tilde{c}_{q}|0\rangle
$$

\footnotetext{
${ }^{1}$ By consistently we mean that the solution must reproduce correctly and unambiguously the value of the D-brane tension.

${ }^{2}$ By identity-based solution we mean a solution which is based on the identity string field $\mathcal{I}$.

${ }^{3}$ Let us remember that the identity string field is the wedge state $\mathcal{I}=U_{1}^{\dagger} U_{1}|0\rangle$. A discussion, needed for the purpose of this paper, about the identity string field can be found in the reference [24].
} 
where $n=0,1,2, \cdots$, and $p, q=1,0,-1,-2, \cdots$ The operators $\hat{\mathcal{L}}^{n}, \hat{\mathcal{B}}$ and $\tilde{c}_{p}$ are defined in [3]. Plugging this ansatz (1.4) into the equation of motion will lead to a system of algebraic equations for the coefficients $f_{n, p}$ and $f_{n, p, q}$. Analyzing these algebraic equations we discover that many of the coefficients can be set to zero, therefore we can use a simpler ansatz than the one given by (1.4), for instance in this paper we are going to use the following ansatz

$$
\Psi=\alpha_{1} c+\alpha_{2} c K+\alpha_{3} K c
$$

where the basic string fields $K$ and $c$ together with $B$ are defined in terms of the identity string field with the appropriated insertions [9, 10]

$$
K \equiv \frac{1}{\pi} \hat{\mathcal{L}} U_{1}^{\dagger} U_{1}|0\rangle, \quad c \equiv U_{1}^{\dagger} U_{1} \tilde{c}_{1}|0\rangle, \quad B \equiv \frac{1}{\pi} \hat{\mathcal{B}} U_{1}^{\dagger} U_{1}|0\rangle
$$

As it will be shown, the solution obtained from the ansatz (1.5) provides ambiguous analytic result for the value of the vacuum energy and consequently for the D-brane tension. In general, it can be shown that the energy of and identity-based solution is of the form $0 \times \infty$ [22], and so it is not well defined. Instead of trying to find a consistent regularization in order to treat our identity-based solution correctly, we are going to show that this particular identity-based solution is equivalent to the recently analytic solution found by Erler and Schnabl [1].

To show the above statement, we need to find an explicit gauge transformation which relates the identity-based solution with the Erler-Schnabl solution. The explicit form of this gauge transformation is given by

$$
\Psi_{\mathrm{E}-\mathrm{S}}=U Q U^{-1}+U \Psi_{I} U^{-1}
$$

where $\Psi_{\mathrm{E}-\mathrm{S}}$ is the Erler-Schnabl solution [1], $\Psi_{I}$ is the identity-based solution, and $U$ is an element of the gauge transformation

$$
U=1+c B K, \quad U^{-1}=1-c B K \frac{1}{1+K} .
$$

Since the Erler-Schnabl solution contains a term depending on $1 /(1+K)$, which is crucial for the computation of the vacuum energy [1], it is not so difficult to guess the form of the gauge transformation 4 which relates our identity-based solution with Erler-Schnabl solution, and in particular the gauge transformation should contain the $1 /(1+K)$ term.

\footnotetext{
${ }^{4}$ Let us remark that in general we can write expressions for $U$ and $U_{-1}$ depending on a function of $K$

$$
U=1+c B f(K), \quad U^{-1}=1-c B \frac{f(K)}{1+f(K)} .
$$
}


Nevertheless we can construct different tachyon vacuum solutions by replacing the term $1 /(1+K)$ with other functions of $K$ satisfying the criteria given in reference [10]. For example the dependence on $K$ in the original Schnabl's solution [3] is given by $-e^{-K}$ [4, 25]. It is clear that if we want to relate our identity-based solution to these different tachyon vacuum solutions we should use another gauge transformation which will depend on the choice of the function of $K$. The reason why we chose the $1 /(1+K)$ dependence is because the computation of the vacuum energy is simplified. It is well known that the case $-e^{-K}$ which corresponds to the original Schnabl's solution has subtleties [3, 4, 25], for instance in order to compute the right value of the vacuum energy the phantom term must be included.

Finally, we carry out the same analysis for the case of the modified cubic superstring field theory, namely we show that a solution based on the identity string field can be brought to the tachyon vacuum solution constructed by Gorbachev using a gauge transformation. Our results show explicitly that how a seemingly trivial identity-based solution can generate a non-trivial configuration which precisely represents to the tachyon vacuum. Certainly it would be very interesting to extend our results to the case of the non-polynomial Berkovits WZW-type superstring field theory.

This paper is organized as follows. In section 2, we review and further develop some properties of the simple analytic solution for tachyon condensation in open bosonic string field theory. We give an example of an identity-based solution which formally solves the equation of motion. After performing a gauge transformation over this seemingly trivial identity-based solution, we obtain Erler-Schnabl's solution which correctly reproduces the value of the D-brane tension. We also show that Erler-Schnabl's solution appears as a particular case of a rather general two-parameter family of solutions. In section 3, we analyze a similar identity-based solution in the modified cubic superstring field theory. As in the bosonic case, by performing a suitable gauge transformation over this identitybased solution, we obtain the known Gorbachev's solution which correctly reproduces the value of the vacuum energy. A two-parameter family of solutions is discussed as well. In section 4, a summary and further directions of exploration are given. The details related to the explicit construction of identity-based solutions and the gauge transformation are left for the appendix.

\section{Simple analytic solution in the bosonic case}

In this section, after review some aspects of the simple analytic solution for tachyon condensation in open bosonic string field theory [1, we analyze a new identity-based solution which formally solves the equation of motion. Since this solution is an isolated identity-like piece, it may happen that the solution is trivial or inconsistent, in fact similar identity-based solutions have been proposed in the past, and for such solutions there is no 
unambiguous analytic calculation for the D-brane tension [22]. However, as we are going to see, after performing a gauge transformation over this seemingly trivial identity-based solution, we obtain the well known Erler-Schnabl's solution which correctly reproduces the value of the D-brane tension. We also show that Erler-Schnabl's solution appears as a particular case of a rather general two-parameter family of solutions.

\section{$2.1 \quad$ Erler-Schnabl's solution}

As derived in Erler-Schnabl's paper [1] using the methods of [3, 10], the simple analytic solution for tachyon condensation in open bosonic string field theory is

$$
\Psi_{\mathrm{E}-\mathrm{S}}=(c+c K B c) \frac{1}{1+K},
$$

where the basic string fields $K, B$ and $c$ are given in the split string notation [4, 9, 10], and they can be written, using the operator representation [3], as follows

$$
\begin{aligned}
K & \rightarrow \frac{1}{\pi} \hat{\mathcal{L}} U_{1}^{\dagger} U_{1}|0\rangle, \\
B & \rightarrow \frac{1}{\pi} \hat{\mathcal{B}} U_{1}^{\dagger} U_{1}|0\rangle, \\
c & \rightarrow U_{1}^{\dagger} U_{1} \tilde{c}(0)|0\rangle .
\end{aligned}
$$

The operators $\hat{\mathcal{L}}, \hat{\mathcal{B}}$ and $\tilde{c}(0)$ are defined in the sliver frame [8] 5 , and they are related to the worldsheet energy-momentum tensor, the $b$ and $c$ ghosts fields respectively, for instance

$$
\begin{aligned}
\hat{\mathcal{L}} & \equiv \mathcal{L}_{0}+\mathcal{L}_{0}^{\dagger}=\oint \frac{d z}{2 \pi i}\left(1+z^{2}\right)(\arctan z+\operatorname{arccot} z) T(z), \\
\hat{\mathcal{B}} & \equiv \mathcal{B}_{0}+\mathcal{B}_{0}^{\dagger}=\oint \frac{d z}{2 \pi i}\left(1+z^{2}\right)(\arctan z+\operatorname{arccot} z) b(z),
\end{aligned}
$$

while the operator $U_{1}^{\dagger} U_{1}$ in general is given by $U_{r}^{\dagger} U_{r}=e^{\frac{2-r}{2}} \hat{\mathcal{L}}$, so we have chosen $r=1$, note that the string field $U_{1}^{\dagger} U_{1}|0\rangle$ represents to the identity string field $1 \rightarrow U_{1}^{\dagger} U_{1}|0\rangle$ [3, 4, 9, 10].

Using the operator representation (2.2)-(2.4) of the string fields $K, B$ and $c$, we can show that these fields satisfy the algebraic relations

$$
\{B, c\}=1, \quad[B, K]=0, \quad B^{2}=c^{2}=0,
$$

and have the following BRST variations

$$
Q K=0, \quad Q B=K, \quad Q c=c K c .
$$

\footnotetext{
${ }^{5}$ Remember that a point in the upper half plane $z$ is mapped to a point in the sliver frame $\tilde{z}$ via the conformal mapping $\tilde{z}=\arctan z$.
} 
As we can see, the solution (2.1) contains explicitly to the identity string field and therefore, we may think that this solution is not well defined in the sense that for such solution there is no unambiguous analytic calculation for the D-brane tension. However, as shown in [1], Erler-Schnabl's solution (2.1) correctly and unambiguously reproduces the value of the D-brane tension. To prove this statement is crucial to write the solution as the following integral

$$
\Psi_{\mathrm{E}-\mathrm{S}}=\int_{0}^{\infty} d t e^{-t}(c+c K B c) \Omega^{t},
$$

this form of the solution is possible since we can invert $1+K$ using the Schwinger parameterization

$$
\frac{1}{1+K}=\int_{0}^{\infty} d t e^{-t(1+K)}=\int_{0}^{\infty} d t e^{-t} \Omega^{t}
$$

As stated in [1], the fact that the solution can be written in terms of a continuous integral over wedge states arbitrarily close to the identity, and not as an isolated identity-like piece, is crucial for the consistency of the solution.

\subsection{The identity-based solution and gauge transformation}

In this subsection, we are going to analyze a new simple identity-based solution to the equation of motion in open bosonic string field theory, this solution is given by

$$
\Psi=c(1-K) \text {. }
$$

Using the algebraic relations (2.7) and (2.8), it is easy to show that the string field $\Psi$ (2.11) satisfies the equation of motion

$$
Q \Psi+\Psi^{2}=0 .
$$

The next step is to compute the value of the vacuum energy, this computation is crucial if we want to verify Sen's first conjecture. Using the equation of motion, the computation of the energy can be reduced to the evaluation of the following correlator

$$
\begin{aligned}
\frac{1}{6 g^{2}}\langle\Psi Q \Psi\rangle & =\frac{1}{6 g^{2}}\langle c(1-K) Q(c(1-K))\rangle \\
& =\frac{1}{6 g^{2}}\left[\left\langle c^{2} K c\right\rangle-\langle c K c K c\rangle+\left\langle c^{2} K c K\right\rangle+\langle c K c K c K\rangle\right] \\
& =0 .
\end{aligned}
$$

The first term and third term vanish because $c^{2}=0$, while the second vanishes for the same reason upon using cyclicity of the correlator. The las term

$$
c K c K c K=c K c \partial c K=c(\partial c)^{2} K
$$


vanishes because $(\partial c)^{2}$. Based on this computation we conclude that the identity-based solution (2.11) seems to be trivial.

Though we have a vanishing result for the value of the vacuum energy, it would be desirable to confirm our calculation by other means, for instance using the $\mathcal{L}_{0}$-level expansion of the identity-based solution (2.11)

$$
\begin{aligned}
\Psi & =U_{1}^{\dagger} U_{1}\left[\tilde{c}_{1}-\frac{1}{\pi} \hat{\mathcal{L}} \tilde{c}_{1}+\frac{1}{2} \tilde{c}_{0}\right]|0\rangle \\
& =\sum_{n=0}^{\infty}\left[\frac{1}{2^{n} n !} \hat{\mathcal{L}}^{n} \tilde{c}_{1}-\frac{1}{\pi} \frac{1}{2^{n} n !} \hat{\mathcal{L}}^{n+1} \tilde{c}_{1}+\frac{1}{2^{n+1} n !} \hat{\mathcal{L}}^{n} \tilde{c}_{0}\right]|0\rangle \\
& =\sum_{n=0}^{\infty} \sum_{p=0}^{1} f_{n, p} \hat{\mathcal{L}}^{n} \tilde{c}_{p}|0\rangle
\end{aligned}
$$

where the coefficient $f_{n, p}$ is given by

$$
f_{n, p}= \begin{cases}\frac{1}{2^{n+1} n !}, & \text { if } n \geq 0 \text { and } p=0 \\ 1, & \text { if } n=0 \text { and } p=1 \\ \frac{1}{2^{n} n !}-\frac{1}{\pi} \frac{1}{2^{n-1}(n-1) !}, & \text { if } n \geq 1 \text { and } p=1 .\end{cases}
$$

Using the $\mathcal{L}_{0}$-level expansion of our identity-based solution (2.15), we find that the value of the vacuum energy is given by

$$
\begin{aligned}
\frac{1}{6 g^{2}}\langle\Psi Q \Psi\rangle & =\frac{1}{6 g^{2}} \sum_{m=0}^{\infty} \sum_{p=0}^{1} \sum_{n=0}^{\infty} \sum_{q=0}^{1} f_{m, p} f_{n, q}\left\langle\operatorname{bpz}\left(\tilde{c}_{p}\right) \hat{\mathcal{L}}^{m+n} Q \tilde{c}_{q}\right\rangle \\
& =-\frac{1}{6 g^{2}}\left[\frac{1}{2}+\frac{2}{\pi^{2}}\right] .
\end{aligned}
$$

Therefore, as we can see, this solution provides ambiguous analytic result for the value of the vacuum energy and consequently for the D-brane tension. It is intriguing to note that even though we have obtained a value different from zero (2.17), this value does not coincide with the one given in equation (1.3). To understand this anomaly better, let us consider two general identity-like string fields with $\mathcal{L}_{0}$ eigenvalues equal to $h_{1}$ and $h_{2}$ respectively

$$
\tilde{\phi}_{1}=U_{1}^{\dagger} U_{1} \tilde{\phi}_{1}(0)|0\rangle, \quad \tilde{\phi}_{2}=U_{1}^{\dagger} U_{1} \tilde{\phi}_{2}(0)|0\rangle,
$$

and compute the correlator $\left\langle\tilde{\phi}_{1}, \tilde{\phi}_{2}\right\rangle$

$$
\begin{aligned}
\left\langle\tilde{\phi}_{1}, \tilde{\phi}_{2}\right\rangle & =\left\langle 0\left|\operatorname{bpz}\left(\tilde{\phi}_{1}(0)\right) U_{1}^{\dagger} U_{1} U_{1}^{\dagger} U_{1} \tilde{\phi}_{2}(0)\right| 0\right\rangle \\
& =\lim _{r \rightarrow 1}\left\langle 0\left|\operatorname{bpz}\left(\tilde{\phi}_{1}(0)\right) U_{r}^{\dagger} U_{r} U_{r}^{\dagger} U_{r} \tilde{\phi}_{2}(0)\right| 0\right\rangle \\
& =\lim _{r \rightarrow 1}\left(\frac{2}{r}\right)^{h_{1}+h_{2}}\left\langle 0\left|\operatorname{bpz}\left(\tilde{\phi}_{1}(0)\right) U_{r} U_{r}^{\dagger} \tilde{\phi}_{2}(0)\right| 0\right\rangle \\
& =\lim _{r \rightarrow 1}\left[\left(\frac{1}{r-1}\right)^{h_{1}+h_{2}}\right]\left\langle 0\left|\operatorname{bpz}\left(\tilde{\phi}_{1}(0)\right) \tilde{\phi}_{2}(0)\right| 0\right\rangle,
\end{aligned}
$$


where we have used the definition of $U_{r}=\left(\frac{2}{r}\right)^{\mathcal{L}_{0}}$. Clearly if the sum of the eigenvalues $h_{1}+h_{2}$ is greater than zero the correlator is divergent, while in the case $h_{1}+h_{2}=0$ the correlator is ambiguous. Only when the sum is less than zero we obtain an unambiguous result $\left\langle\tilde{\phi}_{1}, \tilde{\phi}_{2}\right\rangle=0$. Since the case $h_{1}+h_{2}>0$ could appear in the computation of the vacuum energy, potential singularities can be present. This analysis also shows the origin of the non-triviality of the identity based solution.

In this paper, instead of trying to find a consistent regularization in order to treat our identity-based solution correctly, we are going to show that this particular identity-based solution (2.11) is equivalent to the recently analytic solution found by Erler and Schnabl, to show this statement we need to find an explicit gauge transformation which relates the identity-based solution (2.11) with (2.1), and in fact we have found the explicit form of this gauge transformation

$$
\begin{aligned}
\Psi & =U^{-1}\left(Q+\Psi_{\mathrm{E}-\mathrm{S}}\right) U \\
& =\left[1-c B K \frac{1}{1+K}\right]\left(Q+(c+c K B c) \frac{1}{1+K}\right)[c B K+1] \\
& =\left[1-c B K \frac{1}{1+K}\right]\left(Q(c K B+1)+(c+c K B c) \frac{1}{1+K}(c B K+1)\right) \\
& =\left[1-c B K \frac{1}{1+K}\right]\left(c K c K B-c K^{2}+c \frac{1}{1+K}-c K \frac{1}{1+K} K+c K B c\right) \\
& =\left[1-c B K \frac{1}{1+K}\right]\left(c K c K B-c K^{2}+c \frac{1}{1+K}\left(1-K^{2}\right)+c K B c\right) \\
& =\left[1-c B K \frac{1}{1+K}\right]\left(c K c K B-c K^{2}+c(1-K)+c K B c\right) \\
& =\left[1-c B K \frac{1}{1+K}\right](c B K+1)(c(1-K)) \\
& =c(1-K) .
\end{aligned}
$$

So we just have shown, by explicit computations, that starting with a seemingly trivial identity-based solution (2.11), by a suitable gauge transformation, we arrive to a well behaved solution which correctly reproduce the desire value of the D-brane tension. In the next subsection, we are going to show that the Erler-Schnabl's solution appears as a particular case of a rather general two-parameter family of solutions.

\subsection{Two-parameter family of solutions}

As it was mentioned in the introduction section, in order to find identity-based solutions to the open bosonic string field equations of motion, we should use the following ansatz

$$
\Psi=\sum_{n, p} f_{n, p} U_{1}^{\dagger} U_{1} \hat{\mathcal{L}}^{n} \tilde{c}_{p}|0\rangle+\sum_{n, p, q} f_{n, p, q} U_{1}^{\dagger} U_{1} \hat{\mathcal{B}} \hat{\mathcal{L}}^{n} \tilde{c}_{p} \tilde{c}_{q}|0\rangle
$$


where $n=0,1,2, \cdots$, and $p, q=1,0,-1,-2, \cdots$ The operators $\hat{\mathcal{L}}^{n}, \hat{\mathcal{B}}$ and $\tilde{c}_{p}$ are defined in [3]. Plugging this ansatz (2.21) into the equation of motion will lead to a system of algebraic equations for the coefficients $f_{n, p}$ and $f_{n, p, q}$. Analyzing these algebraic equations we discover that many of the coefficients can be set to zero, therefore we can use a simpler ansatz than the one given by (2.21), for instance we are going to use the following ansatz

$$
\Psi=\alpha_{1} c+\alpha_{2} c K+\alpha_{3} K c,
$$

Plugging this ansatz (2.22) into the equation of motion $Q \Psi+\Psi \Psi=0$, we obtain an algebraic equation for the coefficients $\alpha_{i}$

$$
1+\alpha_{2}+\alpha_{3}=0
$$

and consequently our ansatz (2.22) becomes

$$
\Psi=\alpha_{1} c+\alpha_{2} c K-\left(1+\alpha_{2}\right) K c .
$$

A string field $\Psi^{\prime}$ which identically satisfies the string field equation of motion can be derived by performing a gauge transformation over the identity-based solution (2.24)

$$
\Psi^{\prime}=U(\Psi+Q) U^{-1}
$$

Plugging the expressions (1.8) for the string field $U$ and its inverse $U^{-1}$ into the definition of the gauge transformation (2.25), we obtain a two-parameter family of solutions

$$
\Psi^{\prime}=\left[\alpha_{1}(c+c B K c)+\left(\alpha_{1}+\alpha_{2}\right)(c K+c B K c K)-\left(1+\alpha_{2}\right)(K c+K c B K c)\right] \frac{1}{1+K} .
$$

As it is shown in the appendix, to simplify the calculation of the vacuum energy, we can fix the values of the two parameters $\alpha_{1}$ and $\alpha_{2}$. For instance the particular values of the parameters $\alpha_{1}=1$ and $\alpha_{2}=-1$ correspond to the Erler-Schnabl's solution (2.1). Nevertheless at this point it is interesting to ask: do the solutions with different values of the parameters describe the tachyon vacuum? To provide an answer to this question, we should compute the vacuum energy for the solution (2.26) with arbitrary values for the parameters $\alpha_{1}$ and $\alpha_{2}$.

In order to perform this computation, let us write the solution (2.26) as an expression containing an exact BRST term

$$
\begin{aligned}
\Psi^{\prime}= & {\left[\alpha_{1} c+\left(\alpha_{1}+\alpha_{2}\right) c K-\left(1+\alpha_{2}\right) K c\right] \frac{1}{1+K} } \\
& +Q\left\{\left[\alpha_{1} B c+\left(\alpha_{1}+\alpha_{2}\right) B c K-\left(1+\alpha_{2}\right) K B c\right] \frac{1}{1+K}\right\} .
\end{aligned}
$$


It turns out that the computation of the vacuum energy can be reduced to the evaluation of the following correlator

$$
\frac{1}{6 g^{2}}\left\langle\Psi^{\prime}, Q \Psi^{\prime}\right\rangle=\frac{1}{6 g^{2}}\left\langle\left(a_{1} c+a_{2} c K+a_{3} K c\right) \frac{1}{1+K}\left(a_{1} c K c+a_{2} c K c K+a_{3} K c K c\right) \frac{1}{1+K}\right\rangle,
$$

where we have defined the coefficients

$$
a_{1}=\alpha_{1}, \quad a_{2}=\alpha_{1}+\alpha_{2}, \quad a_{3}=-1-\alpha_{2} .
$$

Expanding the right hand side of equation (2.28), we obtain the following expression for the vacuum energy

$$
\begin{aligned}
& \frac{1}{6 g^{2}}\left[a_{1}^{2}\left\langle c \frac{1}{1+K} c K c \frac{1}{1+K}\right\rangle+a_{1} a_{2}\left\langle c \frac{1}{1+K} c K c K \frac{1}{1+K}\right\rangle+a_{1} a_{3}\left\langle c \frac{1}{1+K} K c K c \frac{1}{1+K}\right\rangle\right. \\
& +a_{2} a_{1}\left\langle c K \frac{1}{1+K} c K c \frac{1}{1+K}\right\rangle+a_{2}^{2}\left\langle c K \frac{1}{1+K} c K c K \frac{1}{1+K}\right\rangle+a_{2} a_{3}\left\langle c K \frac{1}{1+K} K c K c \frac{1}{1+K}\right\rangle \\
& \left.+a_{3} a_{1}\left\langle K c \frac{1}{1+K} c K c \frac{1}{1+K}\right\rangle+a_{3} a_{2}\left\langle K c \frac{1}{1+K} c K c K \frac{1}{1+K}\right\rangle+a_{3}^{2}\left\langle K c \frac{1}{1+K} K c K c \frac{1}{1+K}\right\rangle\right] .
\end{aligned}
$$

All the correlators involved in the evaluation of the vacuum energy (2.30) can be computed using the basic correlator $\left\langle\Omega^{r_{1}} c \Omega^{r_{2}} c \Omega^{r_{3}} c \Omega^{r_{4}}\right\rangle$

$$
\left\langle\Omega^{r_{1}} c \Omega^{r_{2}} c \Omega^{r_{3}} c \Omega^{r_{4}}\right\rangle=\frac{L^{3}}{\pi^{3}} \sin \left[\frac{\pi r_{2}}{L}\right] \sin \left[\frac{\pi r_{3}}{L}\right] \sin \left[\frac{\pi\left(r_{2}+r_{3}\right)}{L}\right], \quad L=r_{1}+r_{2}+r_{3}+r_{4} .
$$

For instance, the expression for the correlator $\left\langle K c \frac{1}{1+K} K c K c \frac{1}{1+K}\right\rangle$ is given by

$$
\begin{aligned}
\left\langle K c \frac{1}{1+K} K c K c \frac{1}{1+K}\right\rangle & =-\left.\int_{0}^{\infty} \int_{0}^{\infty} d t_{1} d t_{2} e^{-t_{1}-t_{2}} \frac{\partial^{3}\left\langle\Omega^{s_{1}} c \Omega^{t_{1}+s_{2}} c \Omega^{s_{3}} c \Omega^{t_{2}}\right\rangle}{\partial s_{1} \partial s_{2} \partial s_{3}}\right|_{s_{1}=0, s_{2}=0, s_{3}=0} \\
& =-\frac{3}{\pi^{2}}
\end{aligned}
$$

Performing similar computations for the rest of the correlators, from (2.30) we get the following expression for the vacuum energy (2.28)

$$
\frac{1}{6 g^{2}}\left\langle\Psi^{\prime}, Q \Psi^{\prime}\right\rangle=-\frac{1}{2 \pi^{2} g^{2}}\left(-a_{1}+a_{2}+a_{3}\right)^{2},
$$

plugging the definition of the coefficients $a_{1}, a_{2}, a_{3}$ (2.29) into the equation (2.33), we remarkably obtain the right value for the vacuum energy

$$
\frac{1}{6 g^{2}}\left\langle\Psi^{\prime}, Q \Psi^{\prime}\right\rangle=-\frac{1}{2 \pi^{2} g^{2}},
$$

therefore this last result (2.34) shows that the two-parameter family of solutions (2.26) describe the tachyon vacuum. 


\section{Simple analytic solution in the superstring case}

In this section, we extend our previous results in order to analyze a new identity-based solution in the modified cubic superstring field theory, and as in the case of open bosonic string field theory, by performing a suitable gauge transformation over this identity-based solution, we obtain the known Gorbachev's solution which correctly reproduces the value of the vacuum energy. A two-parameter family of solutions is discussed as well.

\subsection{The identity-based solution and gauge transformation}

In the superstring case, in addition to the basic string fields $K, B$ and $c$, we need to include the super-reparametrization ghost field $\gamma$ which, in the operator representation, is given by [11]

$$
\gamma \rightarrow U_{1}^{\dagger} U_{1} \tilde{\gamma}(0)|0\rangle
$$

Let us remember that in the superstring case the basic string fields $K, B, c$ and $\gamma$ satisfy the algebraic relations [11, 2 ]

$$
\begin{gathered}
\{B, c\}=1, \quad[B, K]=0, \quad B^{2}=c^{2}=0, \\
\partial c=[K, c], \quad \partial \gamma=[K, \gamma], \quad[c, \gamma]=0, \quad[B, \gamma]=0,
\end{gathered}
$$

and have the following BRST variations

$$
Q K=0, \quad Q B=K, \quad Q c=c K c-\gamma^{2}, \quad Q \gamma=c \partial \gamma-\frac{1}{2} \gamma \partial c
$$

Employing these basic string fields, we can construct the following identity-based solution

$$
\Psi=\left(c+B \gamma^{2}\right)(1-K)
$$

which formally satisfies the equation of motion $Q \Psi+\Psi^{2}=0$, where in this case $Q$ is the BRST operator of the open Neveu-Schwarz superstring theory.

As in the bosonic case, the direct evaluation of the vacuum energy using the identitybased solution (3.4) brings ambiguous result, therefore we should try to find an explicit gauge transformation in order to construct a well behaved solution similar to ErlerSchnabl's solution (2.1). Using the same procedure developed in the previous section, we show that a well behaved solution $\Psi_{\mathrm{G}}$ can be generated from our identity-based solu- 
tion (3.4) by performing a gauge transformation

$$
\begin{aligned}
\Psi & =U^{-1}\left(Q+\Psi_{\mathrm{G}}\right) U \\
& =\left[1-c B K \frac{1}{1+K}\right]\left(Q+\left(c+c K B c+B \gamma^{2}\right) \frac{1}{1+K}\right)[c B K+1] \\
& =\left[1-c B K \frac{1}{1+K}\right]\left(Q(c K B+1)+\left(c+c K B c+B \gamma^{2}\right) \frac{1}{1+K}(c B K+1)\right) \\
& =\left[1-c B K \frac{1}{1+K}\right]\left(c K c K B-c K^{2}+c(1-K)+c K B c+B \gamma^{2}(1-K)\right) \\
& =\left[1-c B K \frac{1}{1+K}\right]\left((c B K+1) c(1-K)+B \gamma^{2}(1-K)\right) \\
& =\left[1-c B K \frac{1}{1+K}\right](c B K+1)\left(\left(c+B \gamma^{2}\right)(1-K)\right) \\
& =\left(c+B \gamma^{2}\right)(1-K) .
\end{aligned}
$$

Remarkably, it turns out that the resulting analytic solution $\Psi_{\mathrm{G}}$ corresponds to the known Gorbachev's solution [2]

$$
\Psi_{\mathrm{G}}=\left(c+c K B c+B \gamma^{2}\right) \frac{1}{1+K}
$$

which, as we are going to verify in the next subsection, correctly reproduces the value of the vacuum energy.

Since the solution (3.6) looks very similar to the bosonic one (2.1), we should write it as a continuous integral over wedge states by inverting $1+K$ and using the Schwinger parameterization (2.10), this way of writing the solution is important if we are interesting in the evaluation of the vacuum energy. Let us mention that the calculation of the vacuum energy for the solution (3.6) was already performed in reference [2] by Gorbachev. Nevertheless, for completeness reasons, we are going to review this important computation in the next subsection.

\subsection{Gorbachev's solution and its vacuum energy}

As it was mentioned in the previous subsection, by inverting $1+K$ and employing the Schwinger parameterization (2.10), we can write the solution (3.6) as the following integral

$$
\Psi_{\mathrm{G}}=\int_{0}^{\infty} d t e^{-t}\left(c+c K B c+B \gamma^{2}\right) \Omega^{t}
$$

now acting the BRST operator $Q$ on the string field $B c: Q(B c)=c K B c+B \gamma^{2}$, we can express (3.7) as

$$
\Psi_{\mathrm{G}}=\int_{0}^{\infty} d t e^{-t} c \Omega^{t}+Q\left[\int_{0}^{\infty} d t e^{-t} B c \Omega^{t}\right]
$$


This way of writing the solution (3.8) is important to simplify the computation of the vacuum energy. Using the equation of motion, the expression for the vacuum energy can be reduced to the evaluation of the following correlator

$$
\begin{aligned}
\frac{1}{6 g^{2}}\left\langle\left\langle\Psi_{\mathrm{G}} Q \Psi_{\mathrm{G}}\right\rangle\right\rangle & =\frac{1}{6 g^{2}} \int_{0}^{\infty} d t_{1} d t_{2} e^{-t_{1}-t_{2}}\left\langle\left\langle c \Omega^{t_{1}}\left(c K c-\gamma^{2}\right) \Omega^{t_{2}}\right\rangle\right\rangle \\
& =\frac{1}{6 g^{2}} \int_{0}^{\infty} d t_{1} d t_{2} e^{-t_{1}-t_{2}}\left[\left\langle Y_{-2} c \Omega^{t_{1}} c K c \Omega^{t_{2}}\right\rangle-\left\langle Y_{-2} c \Omega^{t_{1}} \gamma^{2} \Omega^{t_{2}}\right\rangle\right] \\
& =-\frac{1}{6 g^{2}} \int_{0}^{\infty} d t_{1} d t_{2} e^{-t_{1}-t_{2}}\left\langle Y_{-2} c \Omega^{t_{1}} \gamma^{2} \Omega^{t_{2}}\right\rangle
\end{aligned}
$$

the correlator $\left\langle Y_{-2} c \Omega^{t_{1}} \gamma^{2} \Omega^{t_{2}}\right\rangle$ can be computed using the methods given in [11] 6

$$
\left\langle Y_{-2} c \Omega^{t_{1}} \gamma^{2} \Omega^{t_{2}}\right\rangle=\frac{\left(t_{1}+t_{2}\right)^{2}}{2 \pi^{2}}
$$

plugging the value of the correlator (3.10) into the equation (3.9), we finally obtain

$$
\begin{aligned}
\frac{1}{6 g^{2}}\left\langle\left\langle\Psi_{\mathrm{G}} Q \Psi_{\mathrm{G}}\right\rangle\right\rangle & =-\frac{1}{12 \pi^{2} g^{2}} \int_{0}^{\infty} d t_{1} d t_{2} e^{-t_{1}-t_{2}}\left(t_{1}+t_{2}\right)^{2} \\
& =-\frac{1}{12 \pi^{2} g^{2}}\left(\int_{0}^{\infty} d u u^{3} e^{-u}\right)\left(\int_{0}^{1} d v\right) \\
& =-\frac{1}{2 \pi^{2} g^{2}}
\end{aligned}
$$

where we have used the change of variables defined as follows [1]

$$
\begin{aligned}
u & =t_{1}+t_{2}, \quad u \in[0, \infty), \\
v & =\frac{t_{1}}{t_{1}+t_{2}}, \quad v \in[0,1], \\
d t_{1} d t_{2} & =u d u d v .
\end{aligned}
$$

Note that the value of the vacuum energy obtained in equation (3.11) is in perfect agreement with the value predicted from Sen's first conjecture (1.2). We would like to comment that the value of the vacuum energy can be obtained using another means, for instance using the $\mathcal{L}_{0}$-level expansion of the solution, we have confirmed that the value of the vacuum energy is the same as the one computed analytically (3.11). It should be important to confirm this result by using a third option, namely by employing the usual Virasoro $L_{0}$-level expansion.

\footnotetext{
${ }^{6}$ This correlation function has been computed using the normalization: $\left\langle\xi(x) c \partial c \partial^{2} c(y) e^{-2 \phi(z)}\right\rangle=2$.
} 


\subsection{Two-parameter family of solutions in the superstring case}

Following the same procedures developed in subsection 2.3, in the case of the modified cubic superstring field theory, in the appendix section, we show that Gorbachev's solution (3.6) appears as a particular case of a rather general two-parameter family of solutions

$$
\begin{aligned}
\Psi^{\prime}= & {\left[x_{1} c+x_{2} c K+x_{3} K c+x_{4} B \gamma^{2}+x_{5} B \gamma^{2} K+x_{6} K B \gamma^{2}\right] \frac{1}{1+K} } \\
& +Q\left\{\left[\beta_{1} B c+\left(\beta_{1}+\beta_{2}\right) B c K-\left(1+\beta_{2}\right) K B c\right] \frac{1}{1+K}\right\},
\end{aligned}
$$

where the coefficients $x_{i}$ are given by

$$
\begin{aligned}
& x_{1}=\beta_{1}, \quad x_{2}=\beta_{1}+\beta_{2}, \quad x_{3}=-1-\beta_{2}, \quad x_{4}=1-\beta_{1}, \\
& x_{5}=-\frac{\left(\beta_{1}-1\right)\left(\beta_{1}+\beta_{2}\right)}{\beta_{1}}, \quad x_{6}=\frac{\left(\beta_{1}-1\right)\left(\beta_{2}+1\right)}{\beta_{1}} .
\end{aligned}
$$

Notice that Gorbachev's solution corresponds to the particular values of the parameters $\beta_{1}=1$ and $\beta_{2}=-1$. Nevertheless it is interesting to ask: do the solutions with different values of the parameters describe the tachyon vacuum? To provide an answer to this question, we should compute the vacuum energy for the solution (3.13) with arbitrary values for the parameters $\beta_{1}$ and $\beta_{2}$.

Since the solution (3.13) has an expression containing an exact BRST term, the computation of the vacuum energy can be reduced to the evaluation of the following correlator

$$
\frac{1}{6 g^{2}}\left\langle\left\langle\Psi^{\prime}, Q \Psi^{\prime}\right\rangle\right\rangle=\frac{1}{6 g^{2}}\left\langle\left\langle\Psi_{1}, Q \Psi_{1}\right\rangle\right\rangle
$$

where the string field $\Psi_{1}$ is given by

$$
\Psi_{1}=\left[x_{1} c+x_{2} c K+x_{3} K c+x_{4} B \gamma^{2}+x_{5} B \gamma^{2} K+x_{6} K B \gamma^{2}\right] \frac{1}{1+K} .
$$

After a tedious algebra, by plugging the string field defined in (3.16) into (3.15), we obtain the following expression for the vacuum energy

$$
\begin{aligned}
& \frac{1}{6 g^{2}}\left[-\left\langle\left\langle c \frac{1}{K+1} \gamma^{2} \frac{1}{K+1}\right\rangle\right\rangle x_{1}^{2}-2\left\langle\left\langle c K \frac{1}{K+1} \gamma^{2} \frac{1}{K+1}\right\rangle\right\rangle x_{1} x_{2}-2\left\langle\left\langle c \frac{1}{K+1} K \gamma^{2} \frac{1}{K+1}\right\rangle\right\rangle x_{1} x_{3}\right. \\
& -2\left\langle\left\langle c K \frac{1}{K+1} K \gamma^{2} \frac{1}{K+1}\right\rangle\right\rangle x_{2} x_{3}-\left\langle\left\langle c K \frac{1}{K+1} \gamma^{2} K \frac{1}{K+1}\right\rangle\right\rangle x_{2}^{2}-\left\langle\left\langle K c \frac{1}{K+1} K \gamma^{2} \frac{1}{K+1}\right\rangle\right\rangle x_{3}^{2} \\
& +4\left\langle\left\langle B \gamma^{2} \frac{1}{K+1} c K c \frac{1}{K+1}\right\rangle\right\rangle x_{1} x_{4}+3\left\langle\left\langle B \gamma^{2} K \frac{1}{K+1} c K c \frac{1}{K+1}\right\rangle\right\rangle x_{1} x_{5} \\
& +\left\langle\left\langle B \gamma^{2} \frac{1}{K+1} c K c K \frac{1}{K+1}\right\rangle\right\rangle x_{2} x_{4}+3\left\langle\left\langle B \gamma^{2} \frac{1}{K+1} K c K c \frac{1}{K+1}\right\rangle\right\rangle x_{3} x_{4} \\
& \left.+\left\langle\left\langle K B \gamma^{2} \frac{1}{K+1} c K c \frac{1}{K+1}\right\rangle\right\rangle x_{1} x_{6}\right] .
\end{aligned}
$$


All the correlators involved in the evaluation of the vacuum energy (3.17) can be computed using the basic correlators

$$
\begin{aligned}
\left\langle\left\langle\Omega^{r_{1}} c \Omega^{r_{2}} \gamma^{2} \Omega^{r_{3}}\right\rangle\right\rangle & =\frac{\left(r_{1}+r_{2}+r_{3}\right)^{2}}{2 \pi^{2}}, \\
\left\langle\left\langle\Omega^{r_{1}} B \Omega^{r_{2}} c \Omega^{r_{3}} c \Omega^{r_{4}} \gamma^{2} \Omega^{r_{5}}\right\rangle\right\rangle & =\frac{\left(r_{1}+r_{2}+r_{3}+r_{4}+r_{5}\right) r_{3}}{2 \pi^{2}} .
\end{aligned}
$$

For instance, the expression for the correlator $\left\langle\left\langle B \gamma^{2} \frac{1}{K+1} c K c \frac{1}{K+1}\right\rangle\right\rangle$ is given by

$$
\begin{aligned}
\left\langle\left\langle B \gamma^{2} \frac{1}{K+1} c K c \frac{1}{K+1}\right\rangle\right\rangle & =-\left.\int_{0}^{\infty} \int_{0}^{\infty} d t_{1} d t_{2} e^{-t_{1}-t_{2}} \frac{\partial\left\langle\left\langle B \gamma^{2} \Omega^{t_{1}} c \Omega^{s_{1}} c \Omega^{t_{2}}\right\rangle\right\rangle}{\partial s_{1}}\right|_{s_{1}=0} \\
& =-\frac{1}{2 \pi^{2}} \int_{0}^{\infty} \int_{0}^{\infty} d t_{1} d t_{2} e^{-t_{1}-t_{2}}\left(t_{1}+t_{2}\right) \\
& =-\frac{1}{\pi^{2}}
\end{aligned}
$$

Performing similar computations for the rest of the correlators, from (3.17) we get the following expression for the vacuum energy (3.15)

$$
\frac{1}{6 g^{2}}\left\langle\left\langle\Psi^{\prime}, Q \Psi^{\prime}\right\rangle\right\rangle=\frac{-6 x_{1}^{2}+\left(8 x_{2}+8 x_{3}-4 x_{4}+3 x_{5}+x_{6}\right) x_{1}-2\left(x_{2}+x_{3}\right)^{2}+\left(x_{2}+3 x_{3}\right) x_{4}}{12 g^{2} \pi^{2}},
$$

plugging the definition of the coefficients $x_{1}, x_{2}, x_{3}, x_{4}, x_{5}, x_{6}$ (3.14) into the equation (3.21), we remarkably obtain the right value for the vacuum energy

$$
\frac{1}{6 g^{2}}\left\langle\left\langle\Psi^{\prime}, Q \Psi^{\prime}\right\rangle\right\rangle=-\frac{1}{2 \pi^{2} g^{2}},
$$

therefore this last result (3.22) shows that the two-parameter family of solutions (3.13) describe the tachyon vacuum.

\section{Summary and discussion}

We have studied a new set of identity-based solutions to analyze the problem of tachyon condensation in open bosonic string field theory and cubic superstring field theory, although these solutions seem to be trivial, we have shown that they can be related, by performing a gauge transformation, to well behaved solutions where in the case of open bosonic string field theory, the resulting solution corresponds to the Erler-Schnabl's solution [1, while in the case of the modified cubic superstring field theory corresponds to a similar solution [2] which, unlike the known solutions [11, 13, 14], can be written 
as a continuous integral over wedge states where no regularization or phantom term is required.

Although, after performing the gauge transformation, the resulting Erler-Schnabl-type solutions can be used to compute correctly the value of the vacuum energy, it would be interesting to evaluate directly the vacuum energy using the identity-based solutions, this kind of computations should be possible provide that we can find a consistent regularization scheme. Even though our results suggest that these solutions should reproduce the right value for the D-brane tension, the direct calculation persists as one of the most difficult problems in string field theory [22].

The main motivation of this work was to understand how identity-based solutions can be used to generate well defined solutions which describe to the tachyon vacuum in relatively simple cubic string field theories [16, 17, 18]. It would be important to extend this analysis to the case of Berkovits WZW-type superstring field theory [15], since this theory has a non-polynomial action, the issue for finding the tachyon vacuum solution and the computation of the value of the D-brane tension seems to be highly cumbersome. Nevertheless, we hope that the ideas developed in this paper should be very useful in order to solve this challenging puzzle.

Another important application of the techniques developed in this paper, as mentioned in [26], should be the extension of the subalgebra generated by the basic string fields $K$, $B, c$ and $\gamma$ in order to analyze more general string field configurations [27, 28], such that multiple D-branes, marginal deformations, lump solutions as well as time dependent solutions.

\section{Acknowledgements}

I would like to thank Nathan Berkovits, Ted Erler, Michael Kroyter and Martin Schnabl for useful discussions. This work is supported by CNPq grant 150051/2009-3.

\section{A Derivation of the identity-based solution and gauge transformation}

In this appendix we provide the details related to the derivation of the identity-based solution and the gauge transformation. In the case of open bosonic string field theory, we propose the following ansatz

$$
\Psi=\alpha_{1} c+\alpha_{2} c K+\alpha_{3} K c
$$


plugging this ansatz into the equation of motion $Q \Psi+\Psi \Psi=0$ and using the identity $c K^{2} c=c K c K+K c K c$, we find easily

$$
\alpha_{1}\left(1+\alpha_{2}+\alpha_{3}\right) c K c+\alpha_{2}\left(1+\alpha_{2}+\alpha_{3}\right) c K c K+\alpha_{3}\left(1+\alpha_{2}+\alpha_{3}\right) K c K c=0 .
$$

Therefore, as we can see, the string field equation of motion reduces to the algebraic equation

$$
1+\alpha_{2}+\alpha_{3}=0
$$

and consequently our ansatz (A.1) becomes

$$
\Psi=\alpha_{1} c+\alpha_{2} c K-\left(1+\alpha_{2}\right) K c .
$$

The next step is to perform a gauge transformation over this identity-based solution (A.4). It turns out that the suitable string field $U$ which will be used to define the gauge transformation is given by

$$
U=1+c B K
$$

The inverse of this string field $U$ can be computed using the power series expansion

$$
U^{-1}=\frac{1}{1+c B K}=\sum_{n=0}^{\infty}(-1)^{n}(c B K)^{n}=1+\sum_{n=1}^{\infty}(-1)^{n} c B K^{n}=1-c B K \frac{1}{1+K} .
$$

A string field $\Psi^{\prime}$ which identically satisfies the string field equation of motion $Q \Psi^{\prime}+$ $\Psi^{\prime} \Psi^{\prime}=0$ can be derived by performing a gauge transformation over the identity-based solution (A.4)

$$
\Psi^{\prime}=U(\Psi+Q) U^{-1}
$$

Plugging the expressions (A.5) and (A.6) for the string field $U$ and its inverse $U^{-1}$ into the definition of the gauge transformation (A.7), we obtain

$$
\Psi^{\prime}=\left[\alpha_{1}(c+c B K c)+\left(\alpha_{1}+\alpha_{2}\right)(c K+c B K c K)-\left(1+\alpha_{2}\right)(K c+K c B K c)\right] \frac{1}{1+K} .
$$

To simplify the computation of the vacuum energy, we would like to write ErlerSchnabl-type solution (A.8) in the following way

$$
\Psi^{\prime}=\left[\alpha_{1} c+\chi\right] \frac{1}{1+K}
$$


such that $Q \chi=0$ where $\chi$ is some string field, to satisfy this requirement (A.9) we must impose the following condition on the numerator of equation (A.8)

$$
Q\left[\alpha_{1}(c+c B K c)+\left(\alpha_{1}+\alpha_{2}\right)(c K+c B K c K)-\left(1+\alpha_{2}\right)(K c+K c B K c)\right]=\alpha_{1} c K c
$$

from this last equation (A.10), using the BRST variations (2.8), we obtain

$$
\left(\alpha_{1}+\alpha_{2}\right) c K c K-\left(1+\alpha_{2}\right) K c K c=0,
$$

and therefore the value of the coefficients $\alpha_{1}$ and $\alpha_{2}$ are given by

$$
\alpha_{1}=1 \quad, \quad \alpha_{2}=-1
$$

Finally, plugging the value of these coefficients (A.12) into Erler-Schnabl-type solution (A.8), we get the well known Erler-Schnabl's tachyon vacuum solution in open bosonic string field theory [1]

$$
\Psi_{\mathrm{E}-\mathrm{S}}=[c+c B K c] \frac{1}{1+K}
$$

In the case of the modified cubic superstring field theory, we should use the following ansatz

$$
\Psi=\sum_{n, p} f_{n, p} U_{1}^{\dagger} U_{1} \hat{\mathcal{L}}^{n} \tilde{c}_{p}|0\rangle+\sum_{n, p, q} f_{n, p, q} U_{1}^{\dagger} U_{1} \hat{\mathcal{B}} \hat{\mathcal{L}}^{n} \tilde{c}_{p} \tilde{c}_{q}|0\rangle+\sum_{n, t, u} g_{n, t, u} U_{1}^{\dagger} U_{1} \hat{\mathcal{B}} \hat{\mathcal{L}}^{n} \tilde{\gamma}_{t} \tilde{\gamma}_{u}|0\rangle
$$

where $n=0,1,2, \cdots, p, q=1,0,-1,-2, \cdots$ and $t, u=\frac{1}{2},-\frac{1}{2},-\frac{3}{2}, \cdots$ Plugging this ansatz (A.14) into the equation of motion will lead to a system of algebraic equations for the coefficients $f_{n, p}, f_{n, p, q}$ and $g_{n, t, u}$. Analyzing these algebraic equations we discover that many of the coefficients can be set to zero, therefore we can use a simpler ansatz than the one given by (A.14), namely

$$
\Psi=\beta_{1} c+\beta_{2} c K+\beta_{3} K c+\beta_{4} B \gamma^{2}+\beta_{5} B \gamma^{2} K+\beta_{6} K B \gamma^{2} .
$$

Plugging this ansatz (A.15) into the equation of motion $Q \Psi+\Psi \Psi=0$, we obtain a system of algebraic equations for the coefficients $\beta_{i}$

$$
\begin{aligned}
\beta_{2}+\beta_{3}+1 & =0 \\
-\beta_{2} \beta_{5}+\beta_{3} \beta_{5}-\beta_{5}-\beta_{2} \beta_{6}+\beta_{3} \beta_{6}+\beta_{6} & =0 \\
\beta_{2}-\beta_{3}-\beta_{1} \beta_{5}+\beta_{1} \beta_{6} & =0 \\
\beta_{4}-1 & =0 .
\end{aligned}
$$


Solving this system of algebraic equations, we obtain

$$
\beta_{3}=-\beta_{2}-1, \quad \beta_{4}=1, \quad \beta_{5}=\frac{\beta_{2}}{\beta_{1}}, \quad \beta_{6}=\frac{-\beta_{2}-1}{\beta_{1}},
$$

and consequently our ansatz (A.15) becomes

$$
\Psi=\beta_{1} c+\beta_{2} c K-\left(\beta_{2}+1\right) K c+B \gamma^{2}+\frac{\beta_{2}}{\beta_{1}} B \gamma^{2} K-\frac{\beta_{2}+1}{\beta_{1}} K B \gamma^{2} .
$$

A string field $\Psi^{\prime}$ which identically satisfies the string field equation of motion can be derived, as in the bosonic case, by performing a gauge transformation over the identitybased solution (A.21)

$$
\Psi^{\prime}=U(\Psi+Q) U^{-1}
$$

Plugging the expressions (A.5) and (A.6) for the string field $U$ and its inverse $U^{-1}$ into the definition of the gauge transformation (A.22), we obtain a two-parameter family of solutions

$$
\begin{aligned}
\Psi^{\prime}= & {\left[x_{1} c+x_{2} c K+x_{3} K c+x_{4} B \gamma^{2}+x_{5} B \gamma^{2} K+x_{6} K B \gamma^{2}\right] \frac{1}{1+K} } \\
& +Q\left\{\left[\beta_{1} B c+\left(\beta_{1}+\beta_{2}\right) B c K-\left(1+\beta_{2}\right) K B c\right] \frac{1}{1+K}\right\},
\end{aligned}
$$

where the coefficients $x_{i}$ are given by

$$
\begin{array}{ll}
x_{1}=\beta_{1}, \quad x_{2}=\beta_{1}+\beta_{2}, & x_{3}=-1-\beta_{2}, \quad x_{4}=1-\beta_{1}, \\
x_{5}=-\frac{\left(\beta_{1}-1\right)\left(\beta_{1}+\beta_{2}\right)}{\beta_{1}}, & x_{6}=\frac{\left(\beta_{1}-1\right)\left(\beta_{2}+1\right)}{\beta_{1}} .
\end{array}
$$

These coefficients have been defined to simplify the presentation of our equations. Notice that the Gorbachev's solution [2]

$$
\Psi_{\mathrm{G}}=\left(c+c K B c+B \gamma^{2}\right) \frac{1}{1+K}
$$

corresponds to the particular case $\beta_{1}=1$ and $\beta_{2}=-1$. 


\section{References}

[1] T. Erler and M. Schnabl, A Simple Analytic Solution for Tachyon Condensation, JHEP 0910, 066 (2009), arXiv:0906.0979].

[2] R.V.Gorbachev, New solution of the superstring equation of motion, Theor. Math. Phys. 162, 90 (2010) [Teor. Mat. Fiz. 162, 106 (2010)].

[3] M. Schnabl, Analytic solution for tachyon condensation in open string field theory, Adv. Theor. Math. Phys. 10, 433 (2006), hep-th/0511286.

[4] Y. Okawa, Comments on Schnabl's analytic solution for tachyon condensation in Witten's open string field theory, JHEP 0604, 055 (2006), hep-th/0603159.

[5] E. Fuchs and M. Kroyter, On the validity of the solution of string field theory, JHEP 0605, 006 (2006), hep-th/0603195.

[6] I. Ellwood and M. Schnabl, Proof of vanishing cohomology at the tachyon vacuum, JHEP 0702, 096 (2007), hep-th/0606142.

[7] E. A. Arroyo, Cubic interaction term for Schnabl's solution using Pade approximants, J. Phys. A 42, 375402 (2009), arXiv:0905.2014].

[8] E. Aldo Arroyo, The Tachyon Potential in the Sliver Frame, JHEP 0910, 056 (2009), arXiv:0907.4939].

[9] T. Erler, Split string formalism and the closed string vacuum, JHEP 0705, 083 (2007), hep-th/0611200.

[10] T. Erler, Split string formalism and the closed string vacuum. II, JHEP 0705, 084 (2007), hep-th/0612050.

[11] T. Erler, Tachyon Vacuum in Cubic Superstring Field Theory, JHEP 0801, 013 (2008), arXiv:0707.4591.

[12] I. Y. Aref'eva, R. V. Gorbachev, D. A. Grigoryev, P. N. Khromov, M. V. Maltsev and P. B. Medvedev, Pure Gauge Configurations and Tachyon Solutions to String Field Theories Equations of Motion, JHEP 0905, 050 (2009), arXiv:0901.4533.

[13] I. Y. Aref'eva, R. V. Gorbachev and P. B. Medvedev, Pure Gauge Configurations and Solutions to Fermionic Superstring Field Theories Equations of Motion, J. Phys. A 42, 304001 (2009), arXiv:0903.1273.

[14] I. Y. Aref'eva, R. V. Gorbachev and P. B. Medvedev, Tachyon Solution in Cubic Neveu-Schwarz String Field Theory, Theor. Math. Phys. 158, 320 (2009), arXiv:0804.2017].

[15] N. Berkovits, Super-Poincare Invariant Superstring Field Theory, Nucl. Phys. B 450 (1995) 90, hep-th/9503099.

[16] C. R. Preitschopf, C. B. Thorn and S. A. Yost, Superstring Field Theory, Nucl. Phys. B 337, 363 (1990). 
[17] I. Y. Arefeva, P. B. Medvedev and A. P. Zubarev, New Representation for String Field Solves the Consistency Problem for Open Superstring Field Theory, Nucl. Phys. B 341, 464 (1990).

[18] E. Witten, Noncommutative Geometry And String Field Theory, Nucl. Phys. B 268 (1986) 253.

[19] A. Sen, Descent relations among bosonic D-branes, Int.J.Mod.Phys. A 14 (1999) 4061, hep-th/9902105.

[20] A. Sen, Universality of the tachyon potential, JHEP 9912, 027 (1999), hep-th/9911116.

[21] A. Bagchi and A. Sen, Tachyon Condensation on Separated Brane-Antibrane System, JHEP 0805, 010 (2008), arXiv:0801.3498.

[22] I. Kishimoto and T. Takahashi, Vacuum Structure Around Identity Based Solutions, Prog. Theor. Phys. 122, 385 (2009), arXiv:0904.1095.

[23] I. Ellwood, Singular gauge transformations in string field theory, JHEP 0905, 037 (2009), arXiv:0903.0390.

[24] M. Schnabl, Wedge states in string field theory, JHEP 0301, 004 (2003), hep-th/0201095.

[25] M. Schnabl, Algebraic solutions in Open String Field Theory - a lightning review, arXiv:1004.4858.

[26] S. Zeze, Tachyon potential in KBc subalgebra, arXiv:1004.4351].

[27] M. R. Garousi and E. Hatefi, More on WZ action of non-BPS branes, JHEP 0903, 008 (2009), arXiv:0812.4216.

[28] M. R. Garousi and E. Hatefi, On Wess-Zumino terms of Brane-Antibrane systems, Nucl. Phys. B 800, 502 (2008), arXiv:0710.5875. 